'Universidad Católica de la Santísima Concepción, Oficina de Educación en Ciencias de la Salud (OFECS). Concepción, Chile. ${ }^{2}$ Universidad de Concepción, Departamento de Educación Médica. Concepción, Chile. aprofesor de Biología. Magíster en Ciencias m. Bioquímica. ${ }^{\text {b}}$ Enfermera y Psicóloga. 'Psicólogo. Doctor en Ciencias de la Educación.

Trabajo financiado por el proyecto: Proyecto DIN 02/2016. Dirección de Investigación. Universidad Católica de la Santísima Concepción. Concepción. Chile.

Los autores declaran no tener conflictos de interés.

Recibido el 11 de mayo de 2018, aceptado el 17 de julio de 2019.

Correspondencia a: Marcela Hechenleitner Carvallo Oficina de Educación Médica, Facultad de Medicina, Universidad Católica de la Santísima Concepción. Alonso de Ribera 2850, Concepción, Chile. marcelahc@ucsc.cl

\section{Autoeficacia académica en estudiantes de carreras de la salud de una universidad tradicional chilena}

\author{
MARCELA I. HECHENLEITNER-CARVALLO ${ }^{1, \mathrm{a}}$, \\ ALEJANDRAA. JEREZ-SALINAS ${ }^{1, b}$, CRISTHIAN E. PÉREZ-VILLALOBOS ${ }^{2, c}$
}

\section{Academic self-efficacy among health care undergraduate students in a Chilean university}

Background: Self-efficacy refers to people's expectations about personal resources available for goal achievement. Higher self-efficacy expectations are correlated with higher academic performance. Aim: To analyze the psychometric properties of the Academic Behavior Self-Efficacy Scale (ABSES) and to describe Self-efficacy expectations of students from health-related careers. Material and Methods: A non-probabilistic sample of 479 first- and second-year students from Nursing, Physiotherapy, Medicine, Nutrition and Medical Technology in a public university in Chile, answered the ABSES. Results were analyzed by Exploratory Factor Analysis and its reliability was evaluated using Cronbach's alpha. Also a descriptive analysis and a non-parametric relational analysis were performed. Results: Two factors were identified: Attention and Participation. Attention obtained significantly higher scores than Participation $(p<0.001)$. Compared to their second-year counterparts, first year students had higher scores in Attention $(p<0.001)$ and Participation $(p<0.01)$. Medicine students had higher scores in Participation than students from other careers. Conclusions: $A$ two factor solution was identified for ABSES. Surveyed students had a predominantly passive Self-efficacy, focused in attention. Also, a reduction in selfefficacy was noted among second year students.

(Rev Med Chile 2019; 147: 914-921)

Key words: Delivery of Health Care; Education, Medical; Self Efficacy; Students.
L a autoeficacia hace referencia a las expectativas que tienen las personas sobre su posibilidad de manejar adecuadamente una amplia gama de estresores cotidianos ${ }^{1,2}$. Implica el grado en que estas personas esperan poder organizar y ejecutar acciones para alcanzar el rendimiento deseado ${ }^{1}$. Estas expectativas están influidas por los logros que se han obtenido en el pasado, el desempeño que se ha observado en otros, la persuasión externa, juicios valóricos y estados emocionales ${ }^{1}$.

El concepto de autoeficacia no hace referencia a los recursos que posea el sujeto, sino a como este percibe que puede utilizarlos para conseguir sus objetivos ${ }^{3}$.

La percepción de un alto nivel autoeficacia aumenta las posibilidades de lograr los objetivos, disminuye el estrés frente a las tareas y contribuye a la motivación, lo que influye positivamente en cómo se ven las personas, cómo se sienten y hacen $^{4-6}$. Esto implica que no basta con ser capaz. Para el logro de los objetivos y metas que se propone un individuo, es necesario que este se 
“juzgue capaz” en la utilización de habilidades y destrezas ante diversas situaciones, incluidas las emocionales ${ }^{7}$.

La psicología educativa ha puesto especial atención en el concepto de autoeficacia, generando importantes avances en su comprensión y contribuyendo al mejoramiento de las prácticas pedagógicas ${ }^{8}$. Frente a situaciones académicas es importante tener presente las expectativas de autoeficacia de una persona: un estudiante que ha vivido reiterados fracasos percibe sus propias capacidades de diferente manera que aquél que nunca los ha tenido. Cuanto más fuerte sea la autoeficacia percibida, más retadores son los objetivos que se plantean los sujetos y más firme es su compromiso de alcanzarlos ${ }^{9}$. Se ha identificado la influencia positiva de la autoeficacia en el logro académico en niños y adolescentes ${ }^{10-13}$, siendo un mejor predictor del rendimiento académico que otras variables cognitivas $^{8-19}$ y constituyendo un mediador para los resultados académicos ya que favorece otros procesos cognoscitivos ${ }^{20-21}$. En efecto, la autoeficacia se asociaría directamente a la autoestima y el compromiso académico de los estudiantes ${ }^{22}$ y predeciría sistemáticamente el aprendizaje autodirigido ${ }^{23}$. Además, las creencias de autoeficacia ejercen influencia en la percepción que los estudiantes tienen sobre su capacidad para desempeñar las tareas requeridas, en la elección y metas propuestas, y en el esfuerzo y persistencia de sus acciones encaminadas para lograr sus metas, fomentando patrones de pensamiento y reacciones emocionales positivas ${ }^{24-26}$.

Lo anterior demuestra la importancia de la autoeficacia y su rol como factor determinante en el éxito académico ${ }^{26}$, lo que hace imperante que la educación fortalezca el desarrollo de esta variable en el estudiante fomentando habilidades que le permitan creer en sus propias capacidades ${ }^{16}$. Estudios realizados en estudiantes de Enfermería chilenos han concluido que estrategias como la simulación clínica favorecen el desarrollo de la autoeficacia corroborando una relación positiva entre autoeficacia, motivación y aprendizaje y el desarrollo de habilidades clínicas y comunicacionales ${ }^{27}$.

Se ha documentado que los estudiantes del área de la salud son los que presentan una mayor percepción de autoeficacia en las universidades chilenas $^{28}$. Esto se atribuye a la alta selectividad y exigencias del perfil profesional de estas carreras.
Empero, este grupo de estudiantes está sometido a una gran demanda, competitividad y deterioro psicológico y fisiológico, lo que se ve reflejado en sus altos índice de estrés ${ }^{29,30}$.

Para medir la percepción de autoeficacia académica en la enseñanza superior se han utilizado varios instrumentos que han conseguido identificar el perfil de los estudiantes universitarios en diferentes carreras ${ }^{31,32}$, sin evidencia de validez en Chile. Entre estos, la Escala de Autoeficacia Académica (EACA) de Blanco et al. permite recoger información presente, potencial e ideal ${ }^{31}$.

Ante la falta de estudios psicométricos previos, sobre instrumentos de autoeficacia académica en Chile, el presente estudio ofrece un análisis de su validez y confiabilidad del EACA. Esto, para luego describir la percepción de autoeficacia de los alumnos, en los primeros años de carreras universitarias de la salud y su relación con dos características académicas de éstos: el nivel que cursan y la carrera que estudian.

\section{Método}

El presente estudio utiliza un diseño cuantitativo no experimental, transversal, descriptivo-correlacional.

\section{Participantes}

La muestra de 479 estudiantes fue no-probabilística por cuotas e incluyó alumnos de primer y segundo año de las carreras de Enfermería $(\mathrm{n}=128)$, Medicina $(\mathrm{n}=89)$, Nutrición y Dietética $(n=130)$, Kinesiología $(n=79)$ y Tecnología Médica $(\mathrm{n}=53)$ de la Facultad de Medicina, Universidad Católica de la Santísima Concepción (UCSC). De éstos, 251 (52,4\%) eran de primer año y 228 (47,6\%) de segundo año, $133(27,8 \%)$ eran hombres y $346(72,2 \%)$ mujeres, con una media de edad igual a 19 años $(\mathrm{DE}=1,6)$.

\section{Recolección de datos}

Los estudiantes respondieron la Escala de Autoeficacia Académica (EACA) ${ }^{30}$ junto con un cuestionario sociodemográficos, previo proceso de consentimiento informado, visado por el Comité de Ética de la UCSC. El encuestaje lo realizó presencialmente uno de los investigadores, durante el último mes del semestre académico.

La EACA original consiste en 13 ítems con escala tipo Likert (de $0=$ Nunca a $10=$ Siempre) 
y mide la frecuencia con que actualmente, en forma ideal y si se esfuerza en cambiar, realizaría o manifestaría una acción ${ }^{31}$. Aunque la escala mide escenarios actuales, ideales y potenciales, en esta ocasión los estudiantes sólo respondieron sobre el primer aspecto, indicando la frecuencia con que actualmente realizan una acción o la piensan realizar.

\section{Análisis de datos}

Para la estimación de la validez y confiabilidad de la EACA, se realizó un análisis factorial exploratorio (EFA) aplicando método de extracción de ejes principales (AEP) a fin de evidenciar su validez de constructo. Para analizar la correlación entre factores se usó rho de Spearman y la consistencia interna se calculó con el coeficiente Alfa de Cronbach.

Se realizó un análisis descriptivo y se calcularon las pruebas no paramétricas U de Mann-Whitney para comparar primer y segundo año y la prueba de Kruskal-Wallis para comparar carreras.

Un valor $\mathrm{p}<0,05$ se consideró estadísticamente significativo. El análisis se realizó en el STATA SE 11.0.

\section{Resultados}

\section{Validez y confiabilidad de la EACA}

Primero se evaluó si era pertinente realizar un EFA a los datos. Al hacerlo, se encontró un coeficiente Kaiser-Meyer-Olkin (KMO) de 0,87 y una prueba de esfericidad de Bartlett estadísticamente significativa $(\mathrm{p}<0,001)$, que apoyaban la pertinencia del análisis. Al identificar empíricamente la cantidad de factores, según el criterio de autovalores mayores a 1 , se identificó dos $(4,79$ y 1,08$)$ que eran mayores a $95 \%$ de los factores calculados al azar en 1.000 muestras aleatorias, según el Análisis Paralelo de Horn $(0,30 \text { y } 0,24)^{32}$. Al calcular la matriz de configuración con AEP y rotación oblicua Oblimin directo, el ítem 9 ("Entrego puntualmente los trabajos que se me encargan”) mostró cargas bajo 0,30 , valor mínimo para considerar una carga como significativa ${ }^{32}$, por lo que fue eliminado.

Con los 12 ítems restantes, nuevamente el EFA mostró ser adecuado con un $\mathrm{KMO}=0,87$ y un Bartlett estadísticamente significativo $(p<0,001)$. El criterio de autovalores mayores a 1 , nuevamente identificó empíricamente dos factores $(4,65$ y 1,11 ), mayores al $95 \%$ de los estimados por el Análisis Paralelo $(0,29 \text { y } 0,22)^{32}$. Lo mismo indicó el gráfico de sedimentación (Figura 1).

Su matriz de configuración mostró que todos los ítems presentaban cargas sobre 0,30 , con una carga cruzada en el ítem 8 que fue asignado por afinidad teórica al factor 2 (Tabla 1). De esta forma, los ítems quedaron organizados en dos factores que a partir de un análisis semántico de los ítems incluidos fueron denominados Aten-

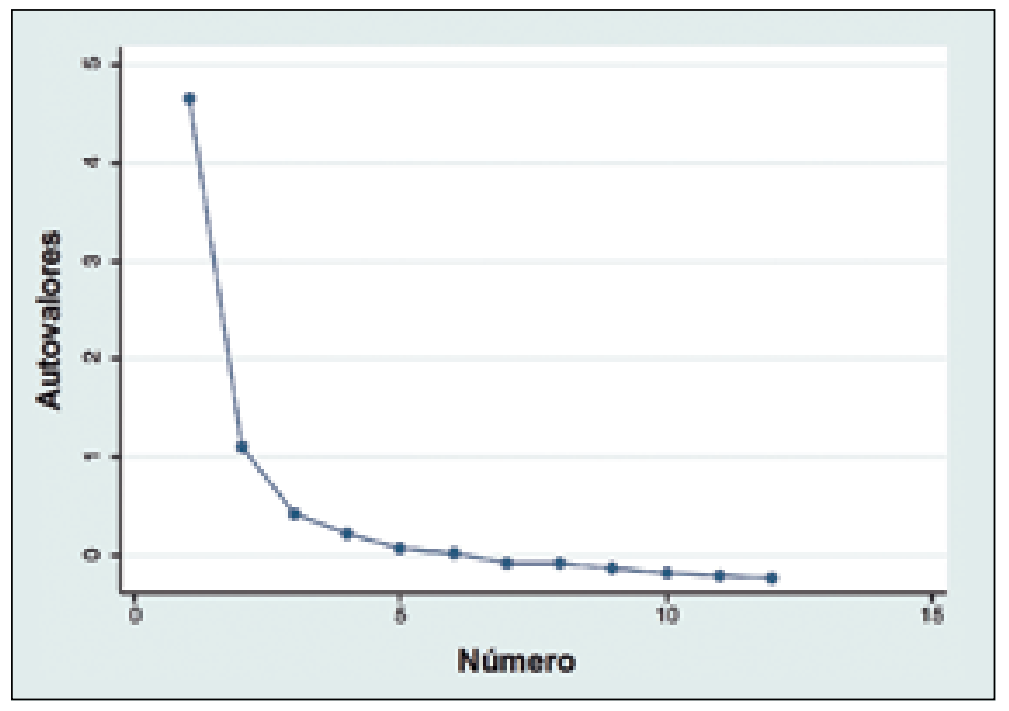

Figura 1. Gráfico de sedimentación para la Escala de Autoeficacia de Conductas Académicas en estudiantes de carreras de la salud (sin ítem 9). 


\section{Tabla 1. Matriz de configuración para la Escala de Autoeficacia de Conductas Académica en estudiantes de carreras de la salud empleando Análisis de Ejes Principales y rotación oblicua Oblimin directo (sin ítem 9)}

\begin{tabular}{|c|c|c|c|c|}
\hline \multicolumn{2}{|c|}{ Ítem } & \multirow[t]{2}{*}{$\mathbf{I}$} & \multirow{2}{*}{$\begin{array}{c}\text { II } \\
0,394\end{array}$} & \multirow{2}{*}{$\begin{array}{c}\text { Comunalidad } \\
\qquad 0,372\end{array}$} \\
\hline 1 & Cumplo con las tareas que se me asignan & & & \\
\hline 2 & Escucho con atención cuando el profesor aclara una duda a un compañero & 0,840 & & 0,608 \\
\hline 3 & Escucho con atención las preguntas y aportaciones de mis compañeros & 0,800 & & 0,613 \\
\hline 4 & Expreso mis ideas con claridad & & 0,722 & 0,546 \\
\hline 5 & Hago comentarios y aportaciones pertinentes & & 0,759 & 0,556 \\
\hline 6 & Pongo atención cuando los profesores dan la clase & 0,755 & & 0,608 \\
\hline 7 & Pongo atención cuando un compañero expone en clases & 0,766 & & 0,573 \\
\hline 8 & $\begin{array}{l}\text { Me preparo para mis exámenes apoyándome en los apuntes de clases, el texto } \\
\text { del curso y las lecturas adicionales }\end{array}$ & 0,324 & 0,334 & 0,336 \\
\hline 10 & Soy cumplido en cuanto a mi asistencia & 0,312 & & 0,168 \\
\hline 11 & Escucho con atención las preguntas y comentarios de mis profesores & 0,735 & & 0,580 \\
\hline 12 & En caso de desacuerdo soy capaz de entablar un diálogo con mis profesores & & 0,616 & 0,364 \\
\hline & $\begin{array}{l}\text { Me siento bien con mi propio desempeño cuando hablo enfrente de una clase } \\
\text { o grupo de gente }\end{array}$ & & 0,680 & 0,438 \\
\hline Aut & tovalor & 4,65 & 1,11 & \\
\hline$\% d$ & de varianza explicada & 80,62 & 18,57 & \\
\hline
\end{tabular}

Fuente: Elaboración propia.

ción y Participación, como se detalla en la Tabla 2. Ambos presentaron una correlación directa (rho $=0,50 ; \mathrm{p}<0,001)$ y sus confiabilidades fueron de 0,84 y 0,80 , respectivamente, calificadas como muy buenas ${ }^{33}$.

\section{Descripción de la autoeficacia de los estudiantes}

Al calcular los puntajes de cada factor, promediando los puntajes de las respuestas a cada ítem dentro de cada factor, variando entre 0 y 10 puntos en ambos casos, se obtuvieron los estadísticos descriptivos de la Tabla 3. Estos muestran asimetría negativa en ambos casos y puntajes cercanos a 10 puntos, lo que muestra que los alumnos tienden a atribuirse alta autoeficacia en ambos factores.

Al comparar los puntajes de ambos factores usando la prueba no-paramétrica $U$ de Mann-Whitney, se encontró diferencias estadísticamente significativas, exhibiendo los alumnos mayor Atención que Participación $(\mathrm{p}<0,001)$ (Tabla 4). Esta misma diferencia se observó tanto en la muestra total como en cada carrera participante.
Adicionalmente, al comparar los puntajes entre carreras, se encontró diferencias estadísticamente significativas entre éstas, en ambos factores. En Atención, los alumnos de Tecnología Médica presentaron niveles más bajos que todas las otras carreras $(\mathrm{p}<0,001)$. En Participación $(\mathrm{p}<0,001)$, ocurrió lo mismo, pero además Medicina tuvo un puntaje significativamente mayor a Enfermería.

Finalmente, se realizó una comparación por nivel formativo, identificándose que los estudiantes de primer año presentaban niveles significativamente superiores de Atención $(\mathrm{p}<0,001)$ y Participación $(\mathrm{p}<0,01)$, que los alumnos de segundo año en la muestra general. Se realizó también este análisis por carrera, exceptuando a Nutrición y Tecnología Médica (por su limitado número de casos), encontrándose que los alumnos de segundo año presentaban menores niveles de Atención en Enfermería ( $p<0,01)$, mientras que los alumnos de primer año presentaban mayor Participación en Enfermería y menor Participación en Medicina en comparación a los alumnos de segundo año (Tabla 5). 
Tabla 2. Factores identificados, definición conceptual e ítems incluidos de la Escala de Autoeficacia de Conductas Académicas en estudiantes de carreras de la salud

\begin{tabular}{|lll|}
\hline Factor & Definición conceptual & \multicolumn{1}{l|}{ Ítems incluidos } \\
\hline Atención & $\begin{array}{l}\text { Se refiere a la confianza que el alumno tiene en su capacidad para mantener } \\
\text { un rol pasivo en sus estudios, atendiendo a docentes y compañeros durante } \\
\text { el proceso de aprendizaje }\end{array}$ & Ítems 2, 3, 6, 7, 10 y 11 \\
\hline Participación & $\begin{array}{l}\text { Se refiere a la confianza que el estudiante reporta sobre su capacidad de tener } \\
\text { un rol activo en sus estudios, aportando a la clase con sus opiniones, cumplien- } \\
\text { do con sus tareas y dialogando con el docente }\end{array}$ & ín, 5,12 y 13 \\
\hline
\end{tabular}

Fuente: Elaboración propia.

Tabla 3. Descriptivos de la Escala de Autoeficacia de Conductas Académicas en estudiantes de carreras de la salud

\begin{tabular}{|lcccccccccc|}
\hline Factor & $\boldsymbol{\alpha}$ & $\mathbf{M}$ & DE & Mín & Máx & P25 & P50 & P75 & Asimetría & Curtosis \\
Atención & 0,84 & 8,57 & 1,22 & 2,50 & 10,00 & 8,00 & 8,83 & 9,50 & $-1,33$ & 5,41 \\
Participación & 0,80 & 7,80 & 1,51 & 2,67 & 10,00 & 6,83 & 8,00 & 9,00 & $-0,72$ & 3,22 \\
\hline
\end{tabular}

$\mathrm{N}=479 ; \alpha=$ Alfa de Cronbach; $\mathrm{M}=$ Media aritmética; $\mathrm{DE}=$ Desviación estándar; Mín = Mínimo; $\alpha=$ Máximo; $\mathrm{P}=$ Percentil. Fuente: Elaboración propia.

Tabla 4. Comparación entre los factores de la Escala de Autoeficacia de Conductas Académicas en estudiantes por carrera y en muestra total

\begin{tabular}{|c|c|c|c|c|c|c|c|}
\hline \multirow[b]{2}{*}{ Carrera } & \multirow[b]{2}{*}{$\mathbf{n}$} & \multicolumn{2}{|c|}{ Atención } & \multicolumn{2}{|c|}{ Participación } & \multirow[b]{2}{*}{$\mathbf{Z}$} & \multirow[b]{2}{*}{$\mathbf{p}$} \\
\hline & & $\mathbf{M}$ & DE & $\mathbf{M}$ & DE & & \\
\hline Enfermería & 128 & 8,63 & 1,27 & 7,58 & 1,61 & 7,57 & $p<0,001$ \\
\hline Kinesiología & 79 & 8,58 & 1,08 & 7,95 & 1,29 & 3,78 & $p<0,001$ \\
\hline Medicina & 89 & 8,85 & 1,03 & 8,22 & 1,48 & 3,90 & $p<0,001$ \\
\hline Nutrición & 130 & 8,72 & 1,14 & 7,98 & 1,48 & 5,81 & $p<0,001$ \\
\hline Tecnología médica & 53 & 7,57 & 1,31 & 6,92 & 1,24 & 2,40 & $p<0,001$ \\
\hline Muestra total & 479 & 8,57 & 1,22 & 7,80 & 1,51 & 11,06 & $p<0,001$ \\
\hline
\end{tabular}

$\mathrm{N}=479$. Fuente: Elaboración propia.

Tabla 5. Comparación por nivel cursado de la percepción de Autoeficacia en estudiantes por carrera y en muestra total

\begin{tabular}{|llcccccc|}
\hline & \multicolumn{9}{c}{ Primer año } & \multicolumn{2}{c}{ Segundo año } & & \\
\multirow{2}{*}{ Atención } & Carrera & $\mathbf{M}$ & $\mathbf{D E}$ & $\mathbf{M}$ & $\mathbf{D E}$ & $\mathbf{Z}$ & $\mathbf{p}$ \\
& Enfermería & 8,83 & 1,34 & 8,43 & 1,17 & 2,69 & $\mathrm{p}<0,01$ \\
& Kinesiología & 8,65 & 1,06 & 8,52 & 1,09 & 0,46 & $\mathrm{p}=0,64$ \\
& Medicina & 8,77 & 0,91 & 8,92 & 1,17 & $-1,45$ & $\mathrm{p}=0,15$ \\
& Muestra total & 8,76 & 1,14 & 8,34 & 1,27 & 4,14 & $\mathrm{p}<0,001$ \\
\multirow{2}{*}{ Participación } & Enfermería & 8,08 & 1,58 & 7,07 & 1,49 & 4,12 & $\mathrm{p}<0,001$ \\
& Kinesiología & 7,75 & 1,30 & 8,04 & 1,30 & $-0,88$ & $\mathrm{p}=0,38$ \\
& Medicina & 7,74 & 1,54 & 8,77 & 1,23 & $-3,37$ & $\mathrm{p}<0,001$ \\
& Muestra total & 7,96 & 1,51 & 7,63 & 1,50 & 2,87 & $\mathrm{p}<0,01$ \\
\hline
\end{tabular}

$\mathrm{N}=$ 479. Fuente: Elaboración propia. 


\section{Discusión}

El análisis factorial exploratorio de la EACA sustenta empíricamente una escala de 12 ítems con una estructura factorial compuesta por dos factores o dimensiones denominados Atención y Participación. Hay que señalar que la dimensión participación incluye las dimensiones de Comunicación y Excelencia de la encuesta original. La consistencia interna de ambas dimensiones es buena, existiendo una alta precisión de medida a pesar del número reducido de ítems que compone la escala.

La dimensión Atención de la autoeficacia indicaría un perfil de estudiante pasivo, que se considera más capaz de recibir información de otros (profesores o pares), que de expresar sus opiniones e inquietudes. Por otro lado, la dimensión Participación, describe un perfil del alumno más activo y protagonista de su aprendizaje, en donde se percibe como capaz de buscar información, resolver sus dudas de manera autónoma con las habilidades que posee. Por tanto, es un alumno que cuenta con más herramientas para el aprendizaje autodirigido ${ }^{34-36}$.

Que el ítem 9 haya sido eliminado, por no relacionarse con los factores identificados, puede deberse a que alude a un requisito básico del desempeño académico (la entrega oportuna de trabajos), volviéndose un ítem de baja exigencia cuyo cumplimiento era una constante y no dependía de la autoeficacia de los estudiantes.

Esto se asocia a que, pese a su heterogeneidad, las carreras de la salud reciben alumnos con los mejores puntajes en la prueba de selección universitaria chilena ${ }^{37}$. Es esperable que el éxito académico requerido para ingresar a estos programas vaya de la mano con un alto nivel de autoeficacia. Esto es respaldado por este estudio, donde ambos factores muestran altos puntajes, destacando la mayor autoeficacia en Medicina ${ }^{1,26}$. Pero es interesante observar que su predominio se encuentra en la Atención más que en la Participación, lo que subraya un perfil académico pasivo.

Lo anterior presenta una alerta para la universidad, pues se contrapone con el modelo formativo de la UCSC, que está basado en el logro de resultados y competencias. Siguiendo este modelo, se espera un desarrollo de la autonomía, resolución de problemas y aprendizaje activo en estos estudiantes al finalizar sus carreras, como resultado de una forma de trabajo que les exige ser el protagonista de su proceso de aprendizaje. Esto debe ser intervenido, atendiendo a una mayor participación de los estudiantes para mejorar su rendimiento, lograr el aprendizaje profundo y permitir al alumno la autorregulación $\mathrm{n}^{27,34,36,38}$. Por este motivo, favorecer el predominio de la Participación por sobre la Atención es una meta que se debe alcanzar durante la formación.

Lo anterior, nos lleva a cuestionar los cambios en la Autoeficacia durante la formación. A diferencia de lo encontrado por Veliz-Burgos y Apodaca $^{28}$, quienes evidenciaron que a mayor año académico mejor percepción de autoeficacia, los alumnos de la muestra general, evidencian en segundo año una menor percepción de autoeficacia que en primero. Esta situación podría darse por la experiencia académica, que pueden hacer más precisos los procesos de autoevaluación, aumentando la autocrítica del estudiante, o a las experiencias negativas que mermarían su percepción de autoeficacia. Por otro lado, existe evidencia, que las carreras del área de la salud están sometidas a alto estrés desde el inicio de su formación ${ }^{29,30}$, y según plantea Bandura el estado emocional influye en cómo es percibida la capacidad de desempeñarse en las tareas propuestas ${ }^{1}$. Por tanto, es posible, que los altos niveles de estrés, a los cuales se ven enfrentados los alumnos, puedan estar influyendo en la disminución de su percepción de autoeficacia.

Sólo en Medicina, la Participación muestra una diferencia opuesta, siendo mayor en segundo año, lo que abre la discusión sobre el efecto que la formación puede tener en la Autoeficacia o como los distintos perfiles de ingreso afectan la evolución de este constructo, sobre todo cuando Medicina es la carrera que ingresa con mayores puntajes de selección en el país ${ }^{37}$.

Nuestros resultados revelaron que dentro del grupo de estudiantes del área de la salud existe diversidad, al menos en la percepción de autoeficacia que presenta cada carrera. Para atender esta diversidad, es que se hace necesario conocer el perfil de los estudiantes y brindar experiencias que favorezcan el desarrollo de conductas que optimicen el aprendizaje en ellos. Es parte del rol de los profesores, informarse acerca del grupo con el cual trabajarán, identificando sus características, de tal manera, de dar a todos, la posibilidad de alcanzar un aprendizaje significativo y profundo. 
Por este motivo, un diagnóstico previo de cada grupo académico es clave: Mientras más información se tenga de los alumnos, mayores serán las posibilidades de atender sus necesidades y lograr alcanzar el perfil de egreso que se declara.

Para finalizar, es necesario considerar que las diferencias identificadas, aunque son estadísticamente significativas, no implican necesariamente diferencias relevantes en la práctica, lo que debe profundizarse en futuros estudios. Adicionalmente, aunque se describe la experiencia en una única universidad de Chile, el estudio permite contar con un instrumento adaptado y con evidencia de validez y confiabilidad en la realidad chilena, lo cual nos permitirá identificar las variables que puedan influir sobre el desempeño de nuestros estudiantes, como es el caso, de la percepción de autoeficacia académica.

Igualmente, este instrumento podrá ser usado para acompañar procesos de intervención que busquen potenciar la autoeficacia de los estudiantes, brindando evidencia de sus efectos.

En esta línea, aunque no existe evidencia de prácticas pedagógicas que fomenten el desarrollo de la autoeficacia en carreras de la salud, existen modelos teóricos como el de Rotter, que desde el concepto de "Locus de control" propone reforzar y valorar las conductas ejecutadas y los resultados que obtienen los individuos ${ }^{39}$. Desde modelos como este, se puede definir planes de mejoramiento en las Escuelas que apoyen esta variable individual, contribuyendo al éxito de los programas formativos y a los procesos de Acreditación.

Agradecimientos: Los autores agradecen a la Dirección de Investigación de la Universidad Católica de la Santísima Concepción, por el apoyo financiero a través del Proyecto DIN 09/2013. También agradecemos a las carreras de la Facultad de Medicina de la UCSC por la disposición para participar de este estudio.

\section{Referencias}

1. Bandura A. Pensamiento y acción: fundamentos sociales. Barcelona: Martínez Roca, S.A. 1987; Pp. 656.

2. Bandura A. Guide for constructing self-efficacy scales. En Pajares F y Urdan T Editores, Self-efficacy beliefs of adolescents. Greenwich, CT, US: Information Age Publishing; 2006; p 307-37.
3. Vinaccia S, Contreras F, Londoño L, Cadena J, Anaya J. Autoeficacia, desesperanza aprendida e incapacidad funcional en pacientes con diagnóstico de artritis reumatoide. Internacional Journal of Clinical and Health Psychology 2005; 5 (1): 129-42.

4. Bandura A. Self-efficacy mechanism in psychobiologic functioning. En Schwarzer R Editores, Self-efficacy: Thought control of action. Washington, D.C, USA: Hemisphere 1992; p 355-94.

5. Bandura A. Self-efficacy. En Manstead ASR \& Hewstone M Editores, Blackwell encyclopedia of social psychology. Oxford, Blackwell, UK 1995; p 453-54.

6. Bandura A. Self-efficacy and health behaviour. En Baum A, Newman S, Wienman J, West R \& McManus C Editores, Cambridge handbook of psychology, health and medicine. Cambridge: Cambridge University Press 1997; p. 160-2.

7. Prieto L. La autoeficacia en el contexto académico. Exploración bibliográfica comentada. Emory University Division of Educational Studies 2003; p 1-14 http:// www.des.emory.edu/mfp/prieto.pdf [Consultado el 3 de mayo de 2018].

8. Pérez E, Lescano C, Zalasar P, Furlám L, Martínez M. Desarrollo y análisis psicométricos de un inventario de autoeficacia para inteligencias múltiples en niños argentinos. Psicoperspectivas 2011; 10 (1): 169-89.

9. Locke EA, Latham GP. A theory of goal setting and task performance. Englewood Cliffs Editores, NJ: Prentice-Hall 1990.

10. Holden G, Moncher M, Schinke S \& Barker K. Self-efficacy of children and adolescents: A meta-analysis. Psychol Rep 1990; 66: 1044-6.

11. Bouffard T, Bouchard M, Goulet G, Denoncourt I, Couture N. Influence of achievement goals and self-efficacy on students' self-regulation and performance. Int J Psychol 2005; 40 (6): 373-84.

12. Contreras F, Espinoza J, Esguerra G, Haikal A, Polonía A, Rodríguez A. Autoeficacia, ansiedad y rendimiento académico en adolescentes. Diversitas 2005; 1 (2): 18394.

13. Hernández LF. Perfil sociodemográfico y académico en estudiantes universitarios respecto a su autoeficacia académica percibida. Psicogente 2018; 21 (39): 35-49.

14. Brown S, Tramayne S, Hoxha D, Telander K, Fan $\mathrm{X}$, Lent R. Social cognitive predictors of college students'academic performance and persistence: a meta-analytic path analysis. J Vocat Behav 2008; 72 (3): 298-308.

15. Schmidt V, Messoulam N, Molina F. Autoconcepto académico en adolescentes de escuelas medias: presentación de un instrumento para su evaluación. Rev Iberoame- 
ricana de Diagnóstico y Evaluación Psicológica 2008; 1 (25): 81-106.

16. Pajares F, Schunk D. Self-Beliefs and school Success: Self-efficacy, self-concept, and school achievement. En Riding R y Rayner S Editores, Perception. Ablex Publishing, London 2001; p 239-66.

17. Pérez E, Cupani M, Ayllón S. Predictores de rendimiento académico en la escuela media: aptitudes, autoeficacia y rasgos de personalidad. Avaliaçao Psicológica 2005; 4 (1): 1-11.

18. Serra-Taylor JA. Autoeficacia y Rendimiento Académico en Estudiantes Universitarios. Rev Griot 2010; 3 (2): 37 45.

19. Véliz A, Dörner A, Sandoval S. Relación entre autoconcepto, autoeficacia académica y rendimiento académico en estudiantes de salud de Puerto Montt, Chile. Rev Educadi 2016; 1 (1): 97-109.

20. Valiante G. Writing Self-efficacy and gender orientation: A developmental perspective, a dissertation proposal. Emory University, Atlanta. 2000.

21. Vera M, Salanova M, Martin del Río B. Self-efficacy among university faculty: how to develop an adjusted scale. Anales de Psicología 2011; 27 (3): 800-07.

22. Pérez MC, Molero MM, Barragán AB, Martos A, Simón MM, Gázquez JJ. Autoeficacia y Engagement en estudiantes de Ciencias de la Salud y su relación con la autoestima. Publicaciones 2018; 48: 193-210.

23. Spormann C, Pérez C, Fasce E, Ortega J, Bastías N, Bustamante C, Ibáñez P. Predictores afectivos y académicos del aprendizaje autodirigido en estudiantes de medicina. Rev Med Chile 2015; 143 (3): 374-82.

24. Pintrich P, García T. Intraindividual differences in students' motivation and self-regulated learning. German journal of educational psychology 1993; 7 (3): 99-107.

25. Bandura A. Self-efficacy: The foundation of agency. En Perrig WJ. \& Grob A. Editores, Control of human behavior, mental processes and consciousness. Mahwak, NJ: Erlbaum 2000; p. 17-33.

26. Canto, Rodríguez JE. Autoeficacia y Educación. Revista Educación y Ciencia. Nueva Época 1998; 2(4): 45-53.

27. Barrios S, Urrutia M, Rubio M. Impacto de la simulación en el desarrollo de la autoeficacia y del locus de control en estudiantes de Enfermería. Educación Médica Superior 2017; 31 (1): 125-36.

28. Véliz-Burgos A, Apodaca P. Niveles de autoconcepto, autoeficacia académica y bienestar psicológico en estu- diantes universitarios de la ciudad de Temuco. Salud \& Sociedad 2012; 3 (2): 131-50.

29. Meyer A, Ramírez L, Pérez C. Percepción de estrés en estudiantes chilenos de Medicina y Enfermería. Rev Educ Cienc Salud 2013; 10 (2): 79-85.

30. Barraza R, Muñoz N, Alfaro M, Álvarez A, Araya V, Villagra J, et al. Ansiedad, depresión, estrés y organización de la personalidad en estudiantes novatos de medicina y enfermería. Rev Chil Neuro-Psiquiatr 2015; 53 (4): 251-60.

31. Blanco H, Martínez M, Zueck M, Gastélum G. Análisis psicométrico de la escala Autoeficacia en conductas académicas en universitarios de primer ingreso. Revista Actualizaciones Investigativas en Educación 2011; 11 (3): 1-26.

32. Martínez R, Hernández M, Hernández M. Psicometría. Madrid: Alianza; 2006.

33. George D, Mallery P. SPSS for Windows step by step: A simple guide and reference. 11.0 update ( $4^{\mathrm{a}} \mathrm{ed}$.). Boston, MA: Allyn \& Bacon. 2003.

34. Fasce E, Pérez C, Ortiz L, Parra P, Matus O. Estructura factorial y confiabilidad de la escala de aprendizaje autodirigido de Fisher, King \& Tague en alumnos de medicina chilenos. Rev Med Chile 2011; 139 (11): 142834.

35. Pérez C, Parra P, Ortiz L, Fasce E. Variables personales $\mathrm{y}$ académicas asociadas al aprendizaje autodirigido en la educación médica. Rev Educ Cienc Salud 2010; 7 (2): 152-9.

36. Márquez C, Fasce E, Pérez C, Ortega J, Parra P, Ortiz L, et al. Aprendizaje autodirigido y su relación con estilos y estrategias de aprendizaje en estudiantes de medicina. Rev Med Chile 2014; 142 (11): 1422-30.

37. Departamento de evaluación, medición y registro educacional (DEMRE) Universidad de Chile. Prueba de Selección Universitaria. Nómina definitiva de carreras, requisitos, vacantes y postulaciones. 2018. http://www. psu.demre.cl/postulacion/carreras-requisitos-vacantes-y-ponderaciones-p2018/ [Consultado el 17 de abril de 2018].

38. Santelices L, Williams C, Soto M, Dougnac A. El efecto de autorregulación del aprendizaje en la enseñanza de conceptos científicos en estudiantes universitarios en ciencias de la salud. Rev Med Chile 2014; 142: 375-81.

39. Rotter J. Generalized Expectancies for Internal Versus External Control of Reinforcement. Psychological Monographs 1966; (609): 1-28. 